\title{
A high-precision null result in the search for rapid oscillations in HD 965
}

\author{
D. W. Kurtz ${ }^{1,2}$, N. Dolez ${ }^{2}$, and M. Chevreton ${ }^{3}$ \\ 1 Centre for Astrophysics, University of Central Lancashire, Preston PR1 2HE, UK \\ ${ }^{2}$ Laboratoire d'Astrophysique de l'OMP, CNRS UMR 5572, Observatoire Midi-Pyrénées, 14 avenue Édouard Belin, \\ 31400 Toulouse, France \\ 3 Observatoire de Paris-Meudon, DAEC, UMR 8632, 92195 Meudon, France
}

Received 2 October 2002 / Accepted 22 November 2002

\begin{abstract}
HD 965 is a strongly magnetic peculiar A star with clear Zeeman splitting in its spectral lines, a rotation period greater than $2 \mathrm{yr}, \mathrm{H}$-line core-wing anomaly, and strongly negative Strömgren $\delta c_{1}$ and $\delta m_{1}$ indices. All of these characteristics are typical of the rapidly oscillating Ap stars, and it has been suggested that HD 965 should be one. We have tested this star for photometric variability under excellent conditions with low scintillation noise using the 2-m TBL for $2.7 \mathrm{hr}$ at Pic du Midi Observatory. The amplitude spectrum of the light curve is flat with noise peaks below $0.2 \mathrm{mmag}$ in the frequency range of the roAp stars. This suggests that it is a non-oscillating Ap star, although proof of that will need further observations each year for many years, to rule out the possibility that rotational modulation has suppressed the observed amplitude in 2002.
\end{abstract}

Key words. stars: chemically peculiar - stars: individual: HD 965 - stars: oscillations - stars: variables: general

\section{Introduction}

In the last two years there have been exciting new theoretical and observational studies of the rapidly oscillating Ap (roAp) stars. Theoretical developments concerning the interaction of pulsation and magnetic fields were published by Cunha \& Gough (2000) and Bigot et al. (2000). The excitation mechanism for roAp stars was studied by Balmforth et al. (2001). Very interesting new work on the interaction of pulsation with both rotation and the magnetic field by Bigot \& Dziembowski (2002) has presented an entirely new look at the oblique pulsator model - they find that the pulsation modes are not axisymmetric modes with pulsation and magnetic axes aligned, as has been believed for the last 20 years. Instead, in their improved oblique pulsator model the pulsation axis is inclined to both the magnetic and rotation axes, and the pulsation modes are complex combinations of spherical harmonics that result in modes that, in many cases, can be travelling waves looking similar to (but are not exactly) sectoral $m$-modes. This new model has already passed some important observational tests, and more are being conducted.

The newest observational results for the roAp stars are spectacular high-resolution spectra of $\gamma$ Equ and HR 3831 (Kochukhov \& Ryabchikova 2001a,b) that show very clearly the extreme stratification effects of abundances and the short vertical wavelength of the pulsation modes in roAp stars.

Send offprint requests to: D. W. Kurtz, e-mail: dwkurtz@uclan.ac.uk
For $\gamma$ Equ $\lambda 6160.24 \AA$ of Pr III and $\lambda 6145.07 \AA$ of Nd III show significant radial velocity variations, while most other lines in a 14 - $\AA$ section of the spectrum show none. A plausible interpretation of this phenomenon is that those ions are concentrated in a thin layer by the effects of radiative diffusion, and that this layer lies near a radial anti-node of the pulsation mode.

This is consistent with previous observations of strong linedepth dependence (atmospheric height dependence) of the pulsation amplitude in the $\mathrm{H} \alpha$ line found by Baldry \& Bedding (2000) for $\alpha$ Cir and by Baldry et al. (1999) for HR 3831, and the strong drop-off of photometric amplitude with increasing wavelength explained by Medupe \& Kurtz (1998). Besides the vertical stratification and the resolution of the vertical structure of the pulsation modes in $\gamma$ Equ and HR 3831, the line profiles shown by Kochukhov \& Ryabchikova (2001a,b) show clearly the signature of travelling waves - probably something similar to $\ell=1, m= \pm 1$. This is qualitatively expected in the improved oblique pulsator model of Bigot \& Dziembowski (2002), but this has yet to be tested quantitatively.

Along with this clear confirmation of the stratification of abundances and proof of the short vertical wavelength of the pulsation modes in roAp stars has come an intriguing suggestion that an observed anomaly in the abundances of $\mathrm{Nd}$ and $\mathrm{Pr}$ determined from their second and third ionisation states may be a signature of roAp stars (Cowley \& Bord 1998; Weiss et al. 2000; Ryabchikova et al. 2001, 2002). These authors have noted that the abundances determined from the second ionisation states of $\mathrm{Nd}$ and $\mathrm{Pr}$ are about 1.5 dex higher than those 
determined from the first ionisation states. This seems likely to be caused by a combination of stratification and temperature structure. In addition to this anomaly Cowley et al. (2001) have noted a core-wing anomaly in the $\mathrm{H}$ lines of a group of five Ap stars. These stars show narrow $\mathrm{H}$-line cores indicative of temperatures near $5500 \mathrm{~K}$, whereas the $\mathrm{H}$-line wings indicate temperatures near $7000 \mathrm{~K}$ to $7500 \mathrm{~K}$. The latter is close to the effective temperatures of these stars, so the H-line cores are strongly indicative of an abnormal T- $\tau$ structure, consistent with the observed abundance stratification. All but one of the five stars studied by Cowley et al. are known roAp stars. The one that is not yet known to be an roAp star is HD 965 .

It is therefore very important to test HD 965 for rapid oscillations characteristic of the roAp stars, as there is, at present, no known parameter that clearly separates roAp from noAp (nonoscillating Ap) stars. Hubrig et al. (2000) have discussed what is known about the roAp stars and noAp stars as groups and find that there is evidence that the noAp stars are somewhat more evolved than the roAp stars, but there is no clear-cut separation between the two groups. Their finding is now complicated by new theoretical work by Cunha (2002) who has calculated an instability strip for roAp stars from models. She finds the more luminous stars have longer periods; the periods for the most unstable modes in her models are as long as 24 min for the most luminous stars found to be unstable. Most searches for roAp stars have used high speed photometry which may fail to find periods longer than about $15 \mathrm{~min}$ because of masking by higher sky transparency noise for these longer periods. Hence we do not have any clear observational separation between the roAp and noAp stars. If HD 965 were to be found to be an roAp star, it would give support to the suggestion that the $\mathrm{Nd}$ and Pr anomalies are characteristic of the group. That would have the practical use of providing an observational signature to find more objects to study, and, much more importantly, would have strong astrophysical implications for the understanding of the interaction of pulsation, rotation and magnetic fields, as well as radiative diffusion and abundance stratification.

It is essentially impossible to prove that any Ap star is an noAp star, i.e. is not pulsating. This is because the roAp stars can be multi-periodic, so that during a typical short photometric observing run, the beating of multiple modes may suppress the amplitude which then goes undetected. In our experience this is unlikely except for very short observing runs of an hour or so. Much more serious is the rotational amplitude modulation that roAp stars undergo. This can lead to time spans of many hours for known roAp stars such as HR 1217, HR 3831 and HD 6532 when no amplitude may be photometrically detectable. For Ap stars with known long rotational periods, such as $\gamma$ Equ with $\mathrm{P}>70 \mathrm{yr}$, there could be years when no amplitude is detectable, yet the star may still be an roAp star.

There is no complete escape from this problem with photometric measurements. New, high spectral resolution, high precision, high time resolution spectroscopic measurements may hold more promise for detecting and studying roAp stars. Mathys et al. (2003) and Kurtz et al. (2003) find, for example, that high resolution VLT spectra of the roAp star HD 166473 show a higher signal-to-noise ratio in radial velocity in the spectra (with precision of $\sim 1 \mathrm{~m} \mathrm{~s}^{-1}$ ) than in the photometry (with a precision of $0.04 \mathrm{mmag}$ ). But it is not practical to test large numbers of stars with VLT-class telescopes; first searches need to be done with telescopes of smaller aperture.

We have thus tested HD 965 for photometric light variations with the 2-m Telescope Bernard Lyot (TBL) at Pic du Midi Observatory. We report here a high-precision null result from a single 2.7-hr run. This is long enough that we are confident that beating from multi-periodicity is unlikely to have masked the presence of rapid oscillations. We cannot rule out the possibility that rotational modulation may have suppressed pulsation that is present, but magnetic measurements of HD 965 suggest a probable rotation period longer than two years (Mathys et al. 1997), so there is little possibility of rotational modulation (if present) bringing up higher amplitude that might be detectable in the current observing season. This star needs to be monitored at least once per observing season for some years to come for a definitive test for photometric variability, and it is an excellent candidate for a very high precision radial velocity study.

HD 965 has a strong magnetic field with Zeeman split components visible in the spectrum. Mathys et al. (1997) give an average surface magnetic field strength of about $4400 \pm 100 \mathrm{G}$. Although they only had a few measurements, the strength decreased monotonically over the two year time-span of their observations. This was the reason for the suggestion that the rotation period is longer than two years - it could be much longer. Cowley et al. (2001) have shown that HD 965 has a strong $\mathrm{H}$ core-wing anomaly similar to four known roAp stars. The Strömgren indices for this star are also typical of the roAp stars: $b-y=0.268, m_{1}=0.296, c_{1}=0.444, \beta=2.740$, $\delta m_{1}=-0.114$ and $\delta c_{1}=-0.216$; the negative $\delta m_{1}$ and $\delta c_{1}$ indices are especially noteworthy - almost all the known roAp stars have negative or strongly negative values of these indices which have been used by Kurtz and Martinez for many years to search for and find most of the known roAp stars.

All of these indications suggest that HD 965 is likely to be an roAp star. The observations presented in this paper indicate that it probably is not.

\section{Observations and analysis}

Photometric observations of HD 965 were obtained for $2.7 \mathrm{hr}$ under excellent photometric conditions using the 2-m TBL of the Pic du Midi Observatory on 2002 August 12/13 beginning at JD 2452499.53542 . We used two channels of the Chevreton four-channel photometer with Johnson $B$ filters, 24 arcsec aperture and 1-s integration times. Sky was monitored continuously in a second channel, although there was no moon and the sky counts were not significant compared to the star counts. Extinction and low-frequency sky transparency variations were removed from the data by fitting a third-order polynomial.

Figure 1 shows an amplitude spectrum of the light curve. We interpret this to be only noise. The highest peak is at $0.877 \mathrm{mHz}(P=19 \mathrm{~min})$ with an amplitude of $0.25 \mathrm{mmag}$. This is slightly longer the known period range of roAp stars, but, as we said in the introduction, the technique of high speed photometry is not best for detecting periods longer than about $15 \mathrm{~min}$, since sky transparency variations increase for longer 


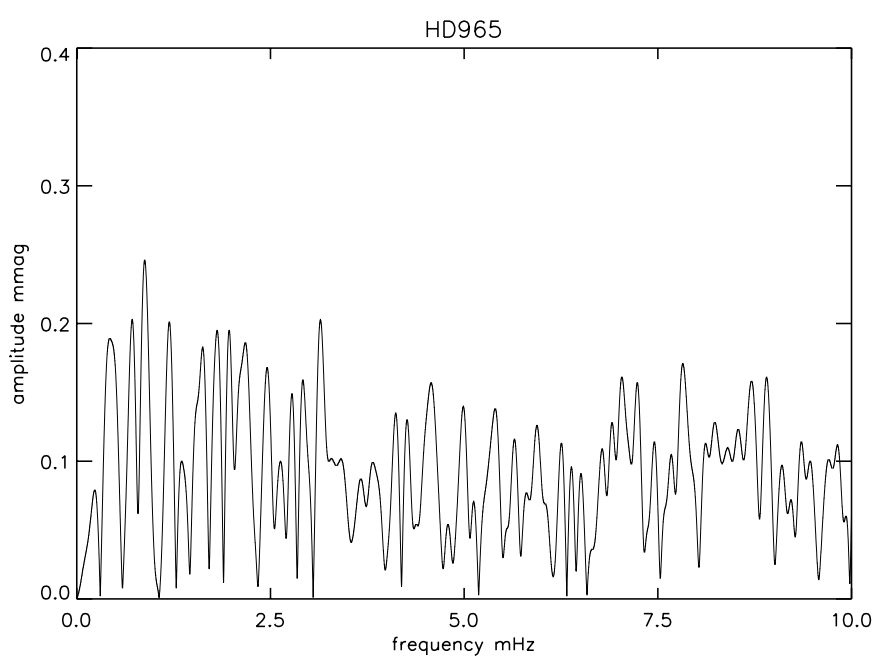

Fig. 1. An amplitude spectrum of the 2.7-hr light curve for HD 965. There is no apparent pulsation in the typical frequency range of roAp stars $(1-3 \mathrm{mHz})$. The highest peak at $0.877 \mathrm{mHz}(P=19 \mathrm{~min})$ is probably noise.

periods (lower frequency) and Cunha (2002) found instability for more luminous roAp stars with periods as long as $24 \mathrm{~min}$. Observationally this is almost unexplored territory. There is only one known star that may be an Ap star and pulsates with periods in the range of Cunha's predicted periods: HD 13038 (Martinez et al. 1999) with pulsation periods of $28 \mathrm{~min}$ and $34 \mathrm{~min}$. Interestingly, there is also a $\delta$ Sct star (not a peculiar star) in an eclipsing binary system, RZ Cas, with a pulsation period of only 23 min (Ohshima et al. 2001; Mkrtichian et al. 2001). The pulsational behaviour of A stars in the $\delta$ Sct instability strip, or the roAp instability strip (if it really exists) in the period range of $15 \mathrm{~min}$ to $30 \mathrm{~min}$ is essentially unstudied observationally.

In any case, we have no reason to believe that the highest peak in Fig. 1 is real. Its amplitude is not significant and photometric noise increases with decreasing frequency. This is simply the highest peak left after our polynomial removal of extinction and sky transparency has removed the lowest frequency amplitude. Indeed, the highest noise peaks in Fig. 1 in the typical frequency range of the known roAp stars $(1-3 \mathrm{mHz})$ are only $0.2 \mathrm{mmag}$. In this frequency range noise is dominated by scintillation, and our use of the $2-\mathrm{m}$ aperture of the TBL is one reason for this low noise level. We consider Fig. 1 to be a strong demonstration that HD 965 has no pulsational amplitude greater than 0.2 mmag at frequencies typical of roAp stars.

\section{Discussion and conclusions}

The characteristics of HD 965 - its strong magnetic field, its H core-wing anomaly, its Strömgren indices - all suggest that it should be an roAp star. We have tested it for $2.7 \mathrm{hr}$ and find no indication of rapid oscillations above $0.2 \mathrm{mmag}$ in amplitude. This is a strong null result. In general, for roAp stars we would recommend the monitoring of a star for several nights, then again several times in an observing season (where telescope time is available - this is more difficult with larger aperture telescopes) to be sure that rotational modulation was not masking light variations, but for HD 965 with its probable rotation period greater than $2 \mathrm{yr}$ (and possibly much greater) it will be necessary to wait until the next observing season to test it again. In this case to get an unambiguous null result will take many years.

HD 965 should be monitored photometrically under excellent conditions and with as large an aperture as possible at least once each year for some years to come. Since it is equatorial, it can be observed from both hemispheres, although the disadvantage of that is that it is always at relatively high airmass where high speed photometry is more susceptible to sky transparency and scintillation noise. More importantly, high resolution, high time resolution spectra should be obtained to search for radial velocity variations, particularly in the Nd and Pr lines. Should this star prove to be an noAp star, then there will still be no clear distinction between the roAp and noAp stars.

\section{References}

Baldry, I. K., \& Bedding, T. R. 2000, MNRAS, 318, 341

Baldry, I. K., Viskum, M., Bedding, T. R., Kjeldsen, H., \& Frandsen, S. 1999, MNRAS, 302, 381

Balmforth, N. J., Cunha, M. S., Dolez, N., Gough, D. O., \& Vauclair, S. 2001, MNRAS, 323, 362

Bigot, L., \& Dziembowski, W. A. 2002, A\&A, 391, 235

Bigot, L., Provost, J., Berthomieu, G., Dziembowski, W. A., \& Goode, P. R. 2000, A\&A, 356, 218

Cowley, C. R., \& Bord, D. J. 1998, ASP Conf. Ser., 143, 346

Cowley, C. R., Hubrig, S., Ryabchikova, T. A., et al. 2001, A\&A, 367, 939

Cunha, M. 2002, MNRAS, 333, 47

Cunha, M., \& Gough, D. O. 2000, MNRAS, 319, 1020

Hubrig, S., Kharchenko, N., Mathys, G., \& North, P. 2000, A\&A, 355, 1031

Kochukhov, O., \& Ryabchikova, T. 2001a, A\&A, 374, 615

Kochukhov, O., \& Ryabchikova, T. 2001b, A\&A, 377, L22

Kurtz, D. W., Martinez, P., Anguma, S., Kalebwe, P., \& Kebede, L. 2003, in preparation

Martinez, P., Ashoka, B. N., Kurtz, D.W., Gupta, S. K., \& Chaubey, U. S. 1999, IBVS, 4677

Mathys, G., Hubrig, S., Landstreet, J. D., Lanz, T., \& Manfroid, J. 1997, A\&AS, 123, 353

Mathys, G., Kurtz, D. W., \& Elkin, V. 2003, in preparation

Medupe, R., \& Kurtz, D. W. 1998, MNRAS, 299, 371

Mkrtichian, D. E., Kusakin, A. V., Gamarova, A. Yu., et al. 2002, ASP Conf. Ser., 256, 259

Ohshima, O., Narusawa, S., Akazawa, H., et al. 2001, AJ, 122, 418

Ryabchikova, T. A., Savanov, I. S., Malanushenko, V. P., \& Kudryavtsev, D. O. 2001, Astron. Rep., 45, 382

Ryabchikova, T. A., Piskunov, N., Kochukhov, O., et al. 2002, A\&A, 384,545

Weiss, W. W., Ryabchikova, T. A., Kupka, F., et al. 2000, ASP Conf. Ser., 203, 487 